\title{
Lattice Boltzmann simulation of vortices merging in a two-phase mixing layer
}

\author{
Y. Y. Yan \& Y. Q. Zu \\ School of the Built Environment, University of Nottingham, UK
}

\begin{abstract}
Mixing layers are commonly observed in flow fields of many types of industrial equipment such as combustion chambers, chemical reactors, and fluid ejectors. It is important to effectively mix two co-flowing gaseous/fluids streams in such equipment. As the mixing of two fluids is an interface and surface tension dominated process, numerical simulations of the mixing process are generally very complex. The present study is concerned with using the lattice Boltzmann method (LBM) to study vertices merging in a two-dimensional two-phase spatial growing mixing layer. Different velocity perturbations are forced at the entrance of the flow field of a rectangular mixing layer; the initial interface between twophases is evenly distributed around the midpoint in a vertical direction. By changing the strength of surface tension and combinations of perturbation waves, the effects of surface tension and velocity perturbation on vortices merging are investigated. Some interesting phenomena, which have not occurred in a singlephase mixing layer, are observed and the corresponding mechanism is discussed. Keywords: lattice Boltzmann method, vortices merging, mixing layer, numerical simulation.
\end{abstract}

\section{Introduction}

Vortices behaviours including the formation and merging are commonly observed in engineering applications, such as in combustion chambers, premixers for gas turbine combustors, chemical lasers, propulsion systems, flow reactors, micro mixers, etc. Controlling the formation and evolution of the coherent structure in the mixing layer can improve efficiency of combustion or chemical reaction processes; therefore studies on vortices behaviour in mixing layers have been carried out both experimentally and computationally. Ho and 
Huang [3] experimentally studied vortices merging in single phase mixing layer. Inoue $[4,5]$ numerically simulated vortices behaviour in double and triple frequency forced two-dimensional spatially growing single-phase mixing layers. By direct numerical simulation (DNS) and large-eddy simulation (LES), Silvestrini [9] have investigated the dynamics of coherent vortices in mixing layer. Lifshitz and Degani [6] proposed mathematical model for turbulent mixing layer with harmonic perturbations; the model reduced numerical complexity, but the surface tension dominated mixing of two-phase fluids was not highlighted.

In the present study, the LBM is employed to simulate vortices behaviour in two immiscible fluids mixing layer. The aims of the study are to understand the effects of surface tension and the perturbation waves on vortices merging, and to obtain an insight to the vortices behaviour at the interface.

\section{The lattice Boltzmann model}

In recent years, the LBM has become an established numerical scheme for simulating multiphase fluid flows. The key idea behind the LBM is to recover the correct macroscopic fluid motion by incorporating the complicated physics into simplified microscopic models or mesoscopic kinetic equations. On simulating multiphase flow problems, four basic LBM models have been reported to date, namely: the chromodynamic model (Andrew et al. [1]), the pseudo-potential model (Shan and Chen [8]), the free energy model (Swift et al. $[9,10])$ and the index function model (He et al. [2]). In this article, the basic method of the index function model for tracking the interface between different fluids is employed. In this model, the velocity and pressure field are given by the distribution function equations of index functions and the pressure, which are given as

$$
\begin{aligned}
& \bar{f}_{\alpha}\left(x+\mathrm{e}_{\alpha} \delta t, t+\delta t\right)-\bar{f}_{\alpha}(x, t)= \\
& \frac{-1}{\tau}\left[\bar{f}_{\alpha}(\mathrm{x}, t)-f_{\alpha}^{(e q)}(\mathrm{x}, t)\right]-\frac{2 \tau-1}{2 \tau} \frac{\left(e_{\alpha}-u\right) \cdot \nabla \psi(\phi)}{R T} \Gamma_{\alpha}(u) \delta t \\
& \bar{g}_{\alpha}\left(\mathrm{x}+\mathrm{e}_{\alpha} \delta t, t+\delta t\right)-\bar{g}_{\alpha}(\mathrm{x}, t)=-\frac{1}{\tau}\left[\bar{g}_{\alpha}(\mathrm{x}, t)-g_{\alpha}^{(e q)}(\mathrm{x}, t)\right]+ \\
& \frac{2 \tau-1}{2 \tau}\left(e_{\alpha}-u\right)\left[\Gamma_{\alpha}(u)(F+G)-\left(\Gamma_{\alpha}(u)-\Gamma_{\alpha}(0)\right) \nabla \psi(\rho)\right] \delta t
\end{aligned}
$$

where $u$ is the macroscopic velocity, $\rho$ the density; $G$ the gravity, and $F$ the surface tension; $T$ the background temperature, $x$ the spatial position vector, and $t$ the time; $\bar{f}_{\alpha}$ is the newly introduced distribution function of index function $\phi$ and $\bar{g}_{\alpha}$ is the newly introduced distribution function of pressure; $f_{\alpha}^{(e q)}$ and $g_{\alpha}^{(e q)}$ are the equilibrium distribution functions in the $\alpha$ th direction and can be expressed respectively as

$$
\begin{aligned}
& f_{\alpha}^{(e q)}(x, t)=\Gamma_{\alpha}(\mathrm{u}) \phi, \\
& g_{\alpha}^{(e q)}(x, t)=\Gamma_{\alpha}(0) p+\left[\Gamma_{\alpha}(\mathrm{u})-\Gamma_{\alpha}(0)\right] \rho R T ;
\end{aligned}
$$


where

$$
\begin{gathered}
\Gamma_{\alpha}(u)=\omega_{\alpha}\left[1+\frac{3}{c^{2}}\left(e_{\alpha} \cdot u\right)+\frac{9}{2 c^{4}}\left(e_{\alpha} \cdot u\right)^{2}-\frac{3}{2 c^{2}} u^{2}\right] ; \\
\omega_{\alpha}= \begin{cases}4 / 9, & \alpha=0 \\
1 / 9, & \alpha=1,3,5,7 \\
1 / 36, & \alpha=2,4,6,8\end{cases}
\end{gathered}
$$

$\delta t$ denotes the length of time step; $p$ the pressure; $\tau$ the dimensionless relaxation time; $\omega_{\alpha}$ is the weighting coefficient; $e_{\alpha}$ the discrete velocity vector, which, in two-dimensional 9-velocity (D2Q9) model, as shown in Fig. 1, is expressed as

$$
\mathrm{e}_{\alpha}= \begin{cases}0, & \alpha=0 \\ \{\cos [(\alpha-1) \pi / 4], \sin [(\alpha-1) \pi / 4]\} c, & \alpha=1,3,5,7 \\ \sqrt{2}\{\cos [(\alpha-1) \pi / 4], \sin [(\alpha-1) \pi / 4]\} c, & \alpha=2,4,6,8\end{cases}
$$

where, $c \equiv \sqrt{3 R T}$ is the lattice constant.

In equations (1) and (2), $\psi(\rho)=p-\rho R T$ to ensure that pressure satisfies the Carnahan-Starling equation:

$$
p=\rho R T \frac{1+b \rho / 4+(b \rho / 4)^{2}-(b \rho / 4)^{3}}{(1-b \rho / 4)^{3}}-a \rho^{2} ;
$$

where, both $a$ and $b$ are equal to $12 R T$ and $R T=1 / 3$; thus,

$$
\psi(\rho)=\rho^{2} \frac{4-2 \rho}{3(1-\rho)^{3}}-4 \rho^{2} .
$$

The index function $\phi$, the macroscopic quantities, $u$ and $p$ are calculated from

$$
\begin{gathered}
\phi=\sum_{\alpha} \bar{f}_{\alpha} \\
p=\sum_{\alpha} \bar{g}_{\alpha}-\frac{1}{2} \mathrm{u} \cdot \nabla \psi(\rho) \delta t \\
\rho R T u=\sum_{\alpha} e_{\alpha} \bar{g}_{\alpha}+\frac{R T}{2}(F+G) \delta t
\end{gathered}
$$

where the surface tension force $F$ is represented as

$$
F=k \phi \nabla \nabla^{2} \phi
$$

where $k$ is the surface tension coefficient, which is related to intermolecular pairwise potential, $u_{\text {att }}$, and can be expressed as $k=-\frac{1}{6} \int_{r>\sigma} r^{2} u_{\text {attr }}(r) d \boldsymbol{r}$, in which $\sigma$ is the effective diameter of molecular; 
The kinematical viscosity $v=(\tau-1 / 2) R T \delta t$ and density $\rho$ are calculated according to the index function:

$$
\begin{aligned}
& \rho(\phi)=\rho_{l}+\frac{\phi-\phi_{l}}{\phi_{h}-\phi_{l}}\left(\rho_{h}-\rho_{l}\right) \\
& v(\phi)=v_{l}+\frac{\phi-\phi_{l}}{\phi_{h}-\phi_{l}}\left(v_{h}-v_{l}\right)
\end{aligned}
$$

where, $\rho_{h}$ and $\rho_{l}$ are the density of heavy and light fluids respectively. $v_{h}$ and $v_{l}$ denote the kinematical viscosity of heavy and light fluids respectively. $\phi_{h}$ and $\phi_{l}$ are the maximum and minimum values of the index function, respectively.

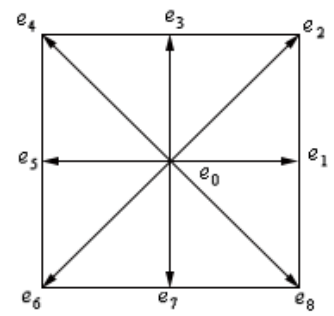

Figure 1: Discrete velocity set of 2-D nine-velocity model

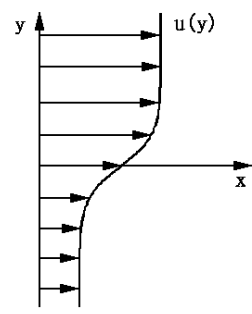

Figure 2: Initial velocity distribution in side the computational domain

\section{Numerical simulation}

To simulate vortices merging in a two-phase spatially growing mixing layer, a rectangular domain of the flow-field is considered as: $D=\left[0, L_{x}\right] \times\left[-L_{y} / 2, L_{y} / 2\right]$, which is surrounded by an inflow boundary at the left, a free outflow boundary at the right, and a slip boundary at two other upper and lower sides. Unless otherwise mentioned, the channel length $L_{x}$ and width $L_{y}$ are set at 250 and 50 respectively. The initial interface between two phases is evenly distributed around the midpoint in vertical direction, the corresponding values of index function are given as $\phi_{h}=0.259$ and $\phi_{l}=0.04$, and the initial velocity field in side the computational domain consists of a hyperbolic tangent profile (see Fig. 2) defined as

$$
u(x, y)=1+R a \cdot \tanh (y / 2) ; v(x, y)=0
$$

The velocity profile at the left boundary $(x=0)$ is given as

$$
u(y)=1+R a \cdot \tanh (y / 2)+u *(y) ; v(y)=v^{*}(y)
$$


where, $u$ and $v$ are the velocity components in the $x$ and $y$ directions, $u^{*}$ and $v^{*}$ are the corresponding velocity perturbations; while, $R a$ is the velocity ratio.

Assuming that $U_{1}$ and $U_{2}$ are the free-stream velocity in the upper and lower layers, respectively, the average velocity, $U=\left(U_{1}+U_{2}\right) / 2$, and the momentum thickness at $x=0$, named as $\theta_{0}$ here, are used as the reference scales for the velocity and length, respectively. Henceforth, all quantities will be normalised with respect to appropriate combinations of $U$ and $\theta_{0}$. Reynolds number for the mixing layer can be defined as $\operatorname{Re}=U \theta_{0} / v$, in which $v$ is kinematical viscosity. It is shown that the influence of Reynolds number upon the kinetic characteristic of the large-scale coherent structures is small enough if Reynolds number is larger than 200. Thus, Reynolds number is set at 200 in the following simulations.

The velocity perturbation $u^{*}$ and $v^{*}$ can be expressed as

$$
\begin{aligned}
u^{*} & =\sum_{n} A_{n} F_{n}^{\prime}(y) \cos \left(\alpha_{n} x-\omega_{n} t+\varphi_{n}\right) / \alpha_{n} \\
v^{*} & =\sum_{n} A_{n} F_{n}(y) \sin \left(\alpha_{n} x-\omega_{n} t+\varphi_{n}\right)
\end{aligned}
$$

where $A, \quad \alpha_{1}, \quad \omega_{1}, \quad \varphi_{1} \quad$ and $\quad A_{i}(i \geq 2), \alpha_{i}(i \geq 2), \omega_{i}(i \geq 2), \varphi_{i}(i \geq 2) \quad$ are amplitudes, wave number, frequency and phase of the basic wave and subharmonic waves, respectively. $F_{n}(y)$ are normalized characteristic mode determined from a linear stability theory. The frequency of basic wave that can lead to the most unstable mode of mixing lay is 0.225 with the velocity ratio $R a=0.5$ (Monkewitz and Patrick, 1982). So, $\omega_{1}$ is given as 0.225 , meanwhile $R a=\left(U_{1}-U_{2}\right) /(2 U)=0.5, \quad F_{n}(y)=1 /\left(1+y^{2}\right), \quad A_{1}=0.02, \quad A_{i}=0.01(i \geq 2)$, $\omega_{2}=\omega_{1} / 2, \omega_{3}=\omega_{1} / 3, \omega_{4}=\omega_{1} / 4, \alpha_{n}=\omega_{n}, \varphi_{1}=0$. In what follows, for simplicity, the subharmonic waves with the frequencies of $\omega_{2}, \omega_{3}$ and $\omega_{4}$ are donated as the second, the third and the fourth subharmonic waves respectively. By superimposing multi-harmonics and changing their phase shifts, one can study the effects of the velocity perturbation to mixing layer.

\section{Results and discussion}

Because the basic wave and several sub-harmonic waves can be forced synchronously, there are many choices of the parameters in equations (18) and (19). Therefore, some typical combinations of perturbation waves are chosen for the simulation. Under some specific initial and boundary conditions, the instabilities will grow and vortices will appear; two or more vortices start to spiral around one another and then merge into a new vortex. The spiralling behaviour and the merging behaviour may repeat itself with the newly formed vortices. In the following, the results of simulation will be visualized by means of plots for phase distribution, vortices contour and corresponding frequency spectrum. 


\subsection{Non-vortex-merging}

In this section, the forced perturbation is only a basic wave with the parameters of $\varphi_{1}$ and $\omega_{1}$. Fig. 3 shows the distributions of two immiscible fluids flow at $\mathrm{t}=350$ for two different values of surface tension. It shows that the vortices appear clearly but no merging takes place under a single-frequency perturbation. This is also be proved by the plots of corresponding frequency spectrum shown in Fig. 4, in which the frequency spectrum for three different cases at $x=120$ is presented. Similar plots are obtained at the other location of the flow-fields, which indicates that the vortices do not experience the merging anywhere.

The effects of surface tension can be judged in Fig. 3. With a zero surface tension, the interface is elongated at upstream. The elongated interface rolls up and forms vortices during the flow. The newly formed vortex spins and migrates continuously towards downstream and sweeps more and more layers of interfaces into it. For $k=0.01$, the flow is qualitatively similar to the zero surface tension case except for the shape of interface in the corresponding vortices; the rollup of the interface in the vortices is slower because the extension of the interface is limited by the surface tension. When $k$ increases to 0.1 , the rolling up of interface at up- and mid-stream is similar to the previous two cases, but the interface evolution is delayed more by the much stronger surface tension. Differences can be identified at the downstream of the mixing layer, where the interface is pinched and broken.

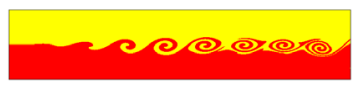

(a) $k=0$

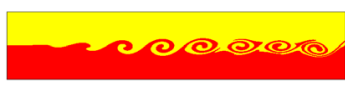

(b) $k=0.01$

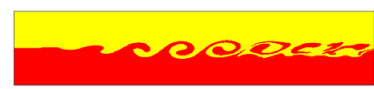

(c) $k=0.1$

Figure 3: Phase distributions with different values of surface tension at $\mathrm{t}=350$.

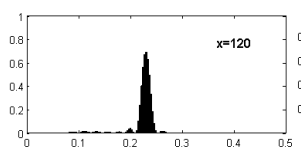

(a) $k=0$

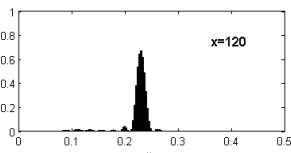

(b) $k=0.01$

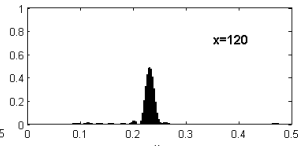

(c) $k=0.1$

Figure 4: Frequency spectrum plots with different values of surface tension at $\mathrm{x}=120$.

\subsection{Two-vortex-merging}

The interaction of the basic wave and its second sub-harmonic wave of $\varphi_{2}=0$ in the mixing layer is first considered. The surface tension parameter, $k$, is chosen as 0 and 0.1 respectively. The phase distribution and corresponding frequency spectrum for these two cases with different surface tension are shown in Figs. 5 and 6 respectively. It can be seen that the vortices formed at the beginning of the mixing layer are nearly of the same frequency as that of the basic wave. Under 
the action of the second sub-harmonic wave, every two of these vortices will wind with each other and merge into a bigger vortex. As shown in Fig. 6, the frequency of the big vortices becomes half of the small ones. The merged vortices start to shear when they move downstream and no further merging takes place. However, with the effect of surface tension, although the rollup of the interface in vortices is slower, the interfacial ends are blunter and the interface pinching and breaking behaviours take place; nevertheless, the basic mode of vortices merging is the same as that of zero surface tension case.

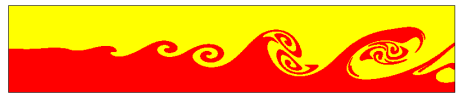

(a) $k=0$

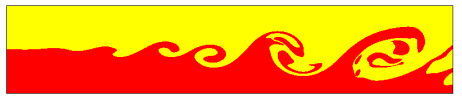

(b) $k=0.1$

Figure 5: Phase distributions with different values of surface tension at $\mathrm{t}=350$.

Fig. 6 shows the frequency spectrum of different surface tension at different position. At $x=40$, the main frequency approximates to $\omega_{1}$, which indicates that no merging takes place. When vortices move to $x=120$, the frequency of $\omega_{1} / 2$ substitutes $\omega_{1}$ to dominate over the flow-field, while the basic frequency, $\omega_{1}$, is still obvious. Such a phenomenon reveals that two vortices are merging at this location and the structures of small vortices have not disappeared completely. Some frequencies which have never been added to the perturbation, such as $3 \omega_{1} / 2$ and $2 \omega_{1}$, can be identified in Fig. 6 . They are the sums or differences of the frequency between the basic wave and sub-harmonic waves. Moreover, the sameness of the frequency spectrum evolution for different cases can further prove that the strength of surface tension does not affect vortices forming, the mode of merging and the migration velocity of the vortices.
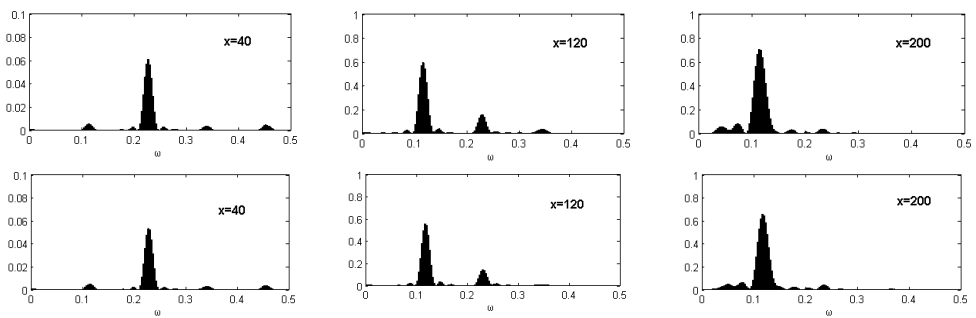

Figure 6: Frequency spectrum plots with different values of surface tension.

\subsection{Three-vortex-merging}

Fig. 7 shows the results in which the perturbation consists of the basic wave and the third sub-harmonic wave of $\varphi_{3}=\pi / 3$. With the interactions of the basic wave and sub-harmonic wave, three vortices merging takes place in the mixing layer. Two downstream vortices merge in the first place, and then the newly 
formed vortex merges with the third one upstream. In fact, as it is shown, no matter what parameter of surface tension is chosen, the basic mode of vortices merging is almost the same. Furthermore, as shown in Fig. 8, the evolutions of frequency spectrum in three cases of different surface tension are quite synchronous.

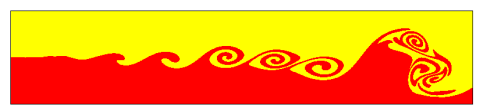

(a) $k=0$

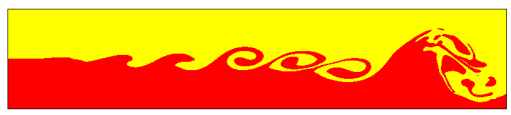

(b) $k=0.1$

Figure 7: Phase distributions with different values of surface tension at $\mathrm{t}=350$.
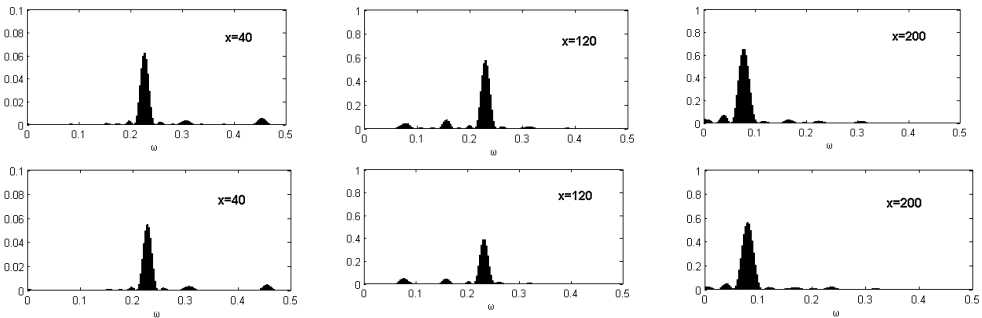

Figure 8: $\quad$ Frequency spectrum plots with different values of surface tension
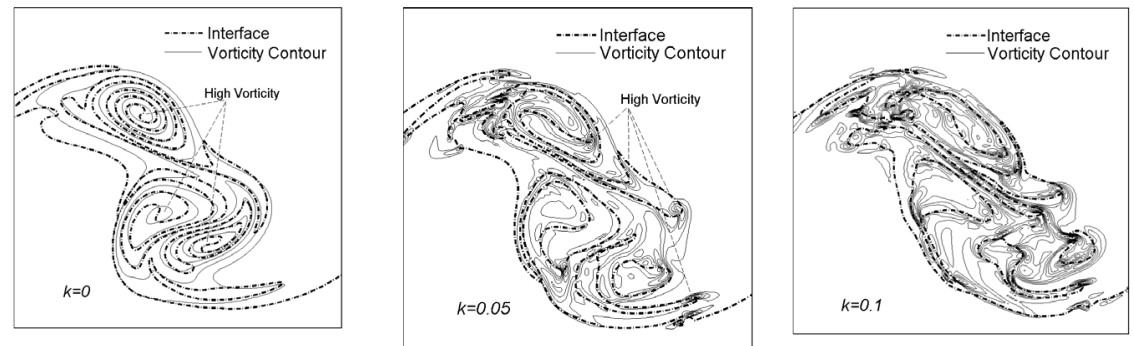

Figure 9: Interface distributions and vortices contours with different surface tension.

Fig. 9 shows a comparison of corresponding vortices contours with the same velocity perturbation and three different values of surface tension within the region of $x \in[200,250]$ at $t=350$; in each case, three main vortices merge into a larger one. It is shown that, with surface tension effect, more small vortices appear in the region of main vortices; and with the increase of surface tension, many small vortices are formed, and the cores of the three main vortices can even disappear. In addition, with zero surface tension at the two fluids interface (indicated by the dished line), the high vortices concentrate in the cores of the vortices; the structures of vortices match the corresponding phase distributions very well. However, with surface tension effect, the vorticity field is disturbed; the vorticity concentrations appear on the interfaces. 


\subsection{More vortex-merging}

Further to the previous discussion, Fig. 10 shows the vortex-merging in a triple-frequency forcing mixing layer. The channel length $L_{x}$ and width $L_{y}$ are given as 375 and 125 respectively. The perturbation consists of a basic wave, a second sub-harmonic wave and a fourth sub-harmonic wave; and all phase shifts equal to zero. Based on this type of perturbation, a phenomenon of four vortices merging takes place in the mixing layer. In the first place, smaller basic vortices are formed upstream in the mixing layer. Thus, every two of these vortices will finish the primary merging and form to a bigger vortex. Then in the flow field downstream, the secondary merging takes place, i.e. every two newly merged vortices repeat the merging process. In this way, the whole process of four-vortexmerging is completed and no further merging behaviour takes place. It is noted from the frequency spectrum plots shown in Fig. 11 that, during the process of vortices merging, the main frequency transfers from $\omega_{1}$ to $\omega_{1} / 4$ via $\omega_{1} / 2$.

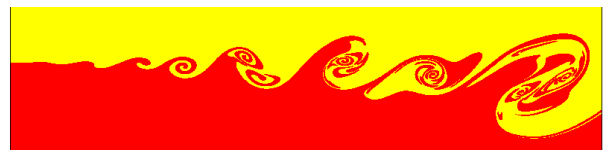

Figure 10: $\quad$ Phase distributions with zero surface tension at $\mathrm{t}=400$.
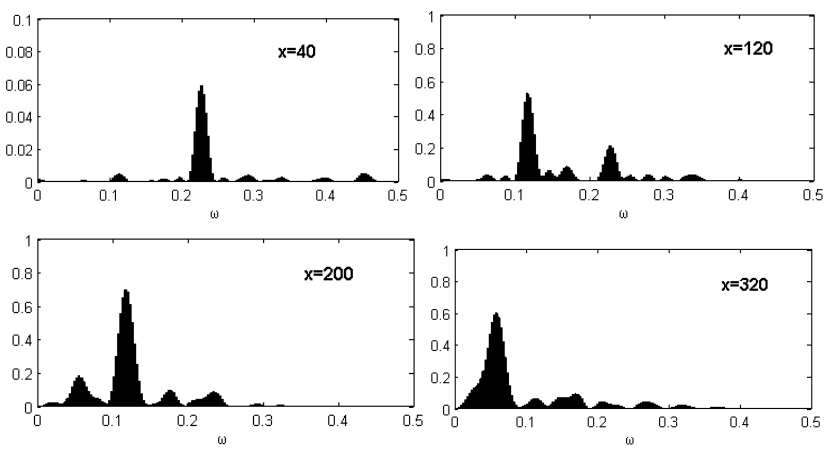

Figure 11: Frequency spectrum plots with zero surface tension.

\section{Conclusion}

The behaviour of vortices merging in two-dimensional two-phase spatially growing mixing layer is numerically studied by the LBM. By changing the strength of surface tension and the combinations of perturbation waves, the effects of the surface tension and the velocity perturbation on the vortices merging are investigated.

With a single-frequency forcing, vortices appear clearly but no merging takes place in the mixing layer. When the mixing layer is forced by the two- or threefrequency perturbation, the vortices start to merge. The results show that the lower frequency of sub-harmonic wave applied, the more vortices are merged. The scale of the large vortex is directly proportional to the number of basic 
vortices being involved in the process. Through vortices merging, the interfacial surface area is enlarged quickly downstream in the flow-field.

In addition, the effect of surface tension strength on vortices formation, the pattern of merging, and the migration velocity of the vortices is limited. Although the rollup of the interface in vortices is slow, the surface tension does have effect on interfacial ends, pinching and breaking. Based on this, it can be assumed that, no matter how strong of the surface tension is, the vortices evolution in two-phase mixing layer should be controlled positively by forcing the suitable perturbation upstream in the flow-field and obtaining expectant flow patterns consequently.

\section{Acknowledgement}

The project is partly supported by Royal Society international joint project Ref. 15127 and British EPSRC under grant EP/D500125/1.

\section{References}

[1] Andrew, K. Gunstensen, Daniel H. Rothman, Stéphane Zaleski and Gianluigi Zanetti. 1991. Lattice Boltzmann model of immiscible fluids. Physical Review A, 43(8): 4320-4327.

[2] He, X., Chen, S. and Zhang, R. 1999. A lattice Boltzmann scheme for incompressible multiphase flow and its application in simulation of Rayleigh-Taylor instability. Journal of Computational Physics, 152(2): 642-663.

[3] Ho C-M., Huang L-S. 1982. Subharmonics and vortex merging in mixing layers. Journal of Fluid Mechanics, 119: 443-473.

[4] Inoue, O., 1992. Double-frequency forcing spatially growing mixing layers. Journal of Fluid Mechanics, 234: 553-581.

[5] Inoue, O., 1995. Note on multiple-frequency forcing on mixing layers, Fluid Dynamics Research, 16(2/3): 161-172.

[6] Lifshitz, Y. and Degani, D., 2004. Mathematical model for turbulent mixing layer with harmonic perturbations. European Congress on Computational Methods in Applied Sciences and Engineering, 1-19.

[7] Monkewitz, P.A. and Patrick Huerre. 1982. Influence of the velocity ratio on the spatial instability of mixing layers. Physics of Fluids, 25(7): 11371143.

[8] Shan, X. and Chen, H. 1993. Lattice Boltzmann model for simulating flows with multiple phases and components. Physical Review E, 47(3): 1815-1819.

[9] Silvestrini, J.H. 2000. Dynamics of coherent vortices in mixing layers using direct numerical and large-eddy simulations. Journal of the Brazilian Society of Mechanical Sciences, 22 (1): 53-67.

[10] Swift, M.R., Osborn, W.R. and J. M. Yeomans. 1995. Lattice Boltzmann simulation of nonideal fluids. Physical Review Letters, 75(5): 830-833.

[11] Swift, M.R., Orlandini, E., W. R. Osborn, and J. M. Yeomans. 1996. Lattice Boltzmann simulations of liquid-gas and binary fluid systems. Physical Review E, 54(5): 5041-5052. 